\title{
The Diffuse Approximation: a derivative tool dedicated to full-field measurements
}

\author{
P. Feissel ${ }^{\mathrm{a}}$ and P. Villon \\ Laboratoire Roberval, UTC, 60200 Compiègne, France
}

\begin{abstract}
This study proposes a filtering tool based on the diffuse approximation, in order to reconstruct strain fields from full-field displacement measurements. The question of the filtering of the noise is adressed and an alternative approach based on space-time filtering is proposed. The methods are then applied to the detection of early damage detection on a tensile test on an interlock composite.
\end{abstract}

\section{Introduction}

The recent development of digitized full-field displacement measurements opens new ways of characterizing materials in solid mechanics [1]. However, for most of the users of these techniques the strain fields rather than the displacement fields provide a real insight into the physics of the material at different scales. Therefore, except for the techniques which provide directly the displacement derivatives, it is necessary to differentiate the data. When the gradients of the displacement fields are relatively low, for example when the materials still behave elastically, the small measurement errors may induce large errors on the computed derivative [2]. So the key work is to develop a stable algorithm, in which it is possible to quantify explicitly the effects induced by noise differentiation.

In a previous collaboration with LMPF-ENSAM Châlons [3], we compared two approaches wich enable to reconstruct the strain while limiting and aiming at controling the effect of the noise. The first approach is based on a global least-squares minimization using Finite-Element shape functions, with local spans as the basis functions [4]. The second approach is based on local weighted least-squares minimization using a polynomial diffuse approximation [5]. Both methods keep some of the treatment local, avoiding large oscillations on the reconstructing fields and yields similar results. Nevertheless, it appeared that the approach based on the Diffuse Approximation did not suffer from mesh dependency of the reconstructed strain and furthermore was more suitable for the choice of the approximation basis and for the fitting of the regularization. Therefore, this method is used in the present study. It is first recalled then applied to the early detection of damage in carbon/epoxy composites.

In the case where a large amount of images are available for a given test, it is possible to take advantage of it by performing the filtering in both space and time. A space-time diffuse approximation filtering is therefore proposed and applied to experimental data, thus compared to the standard spatial Diffuse Approximation.

\section{Reconstruction of the strain field from the displacement field}

\subsection{Framework and notations}

The proposed approach aims at reconstructing the strain field from displacement data, whatever the technique could be to get the latter (DIC or grid method, for example). The measurement zone is

a e-mail: pi erre. fei ssel @itc. fr

This is an Open Access article distributed under the terms of the Creative Commons Attribution-Noncommercial License 3.0, which permits unrestricted use, distribution, and reproduction in any noncommercial medium, provided the original work is properly cited. 
denoted $\Omega$ and the input data are the displacements on a regular grid of data points, denoted $\underline{x}_{i}$. Let the measurements be written as follow:

$$
\underline{\tilde{u}}\left(\underline{x}_{i}\right)=\underline{u}_{e x}\left(\underline{x}_{i}\right)+\delta \underline{u}_{i}\left(\underline{x}_{i}\right) \quad, \forall i \in[1, N]
$$

where $\delta \underline{u}$ represents the perturbation on the measurements and $\underline{u}_{e x}$ is the exact mechanical field. The aim of the reconstruction is to yield a strain field as close as possible to the one associated with $\underline{u}_{e x}$.

The reconstruction operator being linear (see 2.2), one can split the reconstruction of $\underline{\tilde{u}}$ as the sum of the reconstruction of the exact field $\underline{u}_{e x}$ and the one of the perturbation alone. The reconstructed field $\underline{u}_{a p}(\underline{x})$, at any $\underline{x}$, can therefore be written as:

$$
\underline{u}_{a p}(\underline{x})=\underline{u}_{e x}(\underline{x})+\delta \underline{u}_{k}(\underline{x})+\delta \underline{u}_{b}(\underline{x})
$$

where $\delta \underline{u}_{k}(\underline{x})$ is the approximation error due to the filtering of the exact field and $\delta \underline{u}_{b}(\underline{x})$ is the random error on the reconstruction. The same splitting can be applied to the reconstructed strain field $\epsilon_{a p}$.

\subsection{The Diffuse Approximation (DA) as a filtering tool}

The proposed approach is based on the use of local weighted least-squares [6]. Here, the local regression tool is the Diffuse Approximation [5], who was first developed for the solving of partial differential equations. The parameter controling the filtering is the the span of the influence zone of each data point. One keypoint of the method is that it yields both a continuous displacement field and its derivatives (in a diffuse way) at ones, as explained in the following.

The reconstructed field is sought at any point of $\Omega$, as the solution of the following minimization problem:

$$
\min _{a(\underline{x})} \frac{1}{2}(P\{a\}-\widetilde{U})^{T} W(P\{a\}-\widetilde{U}) \quad \text { with, } \quad P=\left[\begin{array}{c}
p\left(\underline{x}-\underline{x}_{1}\right) \\
\ldots \\
p\left(\underline{x}-\underline{x}_{N}\right)
\end{array}\right]_{i \in V(\underline{x})}
$$

where $p(x)$ is the line vector of the monomials of the approximation basis, which is not to be necessarily polynomial. Here, polynomial basis of degree 2 is chosen, because, it appeared in [7] it was a good compromise between filtering and approximation error. With such a basis, the terms $a_{2}(\underline{x})$ and $a_{3}(\underline{x})$ represent the first order derivatives at point $\underline{x}$ in a diffuse way. $V(\underline{x})$ represents the nieghbourhood of $x$ collecting the data points $\underline{x}_{i}$ taken into account in the reconstruction at point $\underline{x}$. The matrix $W$ is a diagonal matrix made of the weighting functions $w\left(\underline{x}, \underline{x}_{i}\right)$ which ensure the continuity of the solution. The weighting functions are such that they equal zero outside the neighbourhood $V(\underline{x})$, whose size is described by the span $R$.

\subsection{Theorical filtering of the noise}

The minimization problem of the quadratic criterium (3) leads to the solving of a linear system. This means, as suggested above, that one can study individually the filtering of the noise and the approximation error related to the use of too large influence span $R$.

Denoting $M_{\epsilon}$ the strain reconstruction operator and assessing the perturbation is a white gaussian noise sample with known standard deviation $\gamma$, the covariance matrix of the reconstructed strain can be characterized as follow:

$$
\operatorname{COV}\left(\delta \epsilon_{b}\right)=\gamma^{2} M_{\epsilon} M_{\epsilon}^{T}
$$

Figure 1 presents the ratio of the standard deviation of $\epsilon_{X X}$ to the standard deviation of the measurements noise as a function of the span $R$ and the offset of the reconstruction point with respect to its neighbourhood $V(\underline{x})$ (a non-zero offset would occur when reconstructing the strain close to an edge of the data area). The increase of the span $R$ improves the filtering and the offset implies a larger span $R$ in order to keep the same filtering quality. 


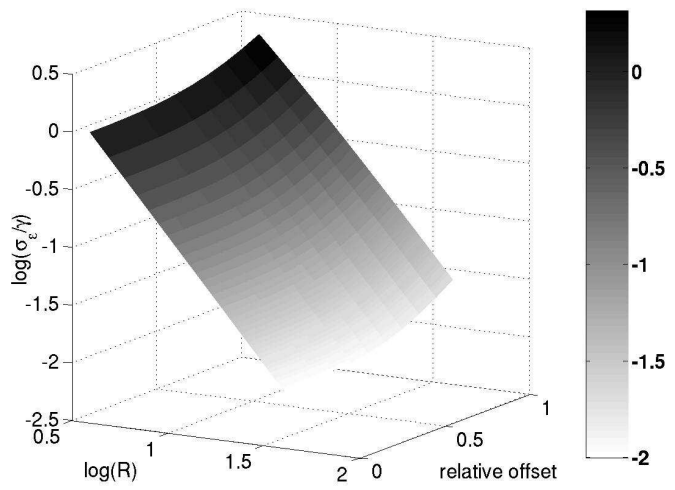

Fig. 1. Standard deviation on $\epsilon_{X X}$ as a function of the span $R$ and the offset, logscale

The reconstruction operator $M$ can actually be split in two parts, one dimensionless part that depends on the spatial distribution of the data points (mainly their number) and one part introducing the physical size of the neighbourhood, related to $R$. It writes as follows:

$$
M=D_{R}^{-1} M_{a d i} \Rightarrow \operatorname{cov}(\{\delta a\})=\sigma_{b}^{2} D_{R}^{-1} \underbrace{M_{a d i} M_{a d i}^{T}}_{A} D_{R}^{-1}
$$

where $M_{a d i}$ and $A$ take the position and number of points of the neighbourhood into account. $D$ deals with the physical size of the neighbourhood, related to $R$.

The variances on the displacement and its first derivative are hence given by:

$$
\begin{aligned}
& \text { - displacement - order } 0 \text { field : } \operatorname{cov}\left(a_{1}\right)=A_{11} \\
& \text { - strain - order } 1 \text { field : } \operatorname{cov}\left(a_{2}\right)=\frac{1}{R^{2}} A_{22}
\end{aligned}
$$

The terms of the matrix $A$ tend to 0 almost as the inverse of the number of points implied in the reconstruction. In a practical case, as the radius $R$ increases, both the physical size of the neighbourhood and the number of points increase, $A$ and $D$ are hence both modified and tend to 0 . One can note that the filtering rate with respect to $R$ on the derivative is larger than on the displacement itself (but its initial value is also larger).

\section{Space-Time Diffuse Approximation filtering}

In the case where a large amount of images are available for a given test, it is possible to improve the spatial resolution of the reconstructed strain by filtering on space and time at once. The measured displacement fields are therefore stacked together along time, so that we now seek a diffuse field of a three-dimensionnal variable $X=(\underline{x}, t)$. The approximated field is written as follow:

$$
u_{a p}(\underline{X})=p(\underline{X})^{T} a(\underline{X}) \quad \text { with, } \quad \underline{X}=(\underline{x}, t)
$$

The weighting function defining the neighbourhoods are simply extended to the space-time framework:

$$
w\left(\underline{X}, \underline{X}_{i}\right)=w_{r e f}\left(\frac{x-x_{i}}{R_{x}}\right) w_{r e f}\left(\frac{y-y_{i}}{R_{y}}\right) w_{r e f}\left(\frac{t-t_{i}}{R_{t}}\right)
$$

The local weighted least-squares are therefore formulated on space and time and one can tune both a span in space, $R_{x}$ and a span in time, $R_{t}$. The characterization of the effect of the couple $\left(R_{x}, R_{t}\right)$ 
on the filtering of the noise on the measurements can be studied from a theoretical point of view, as presented in Section 2.3 for the 2D diffuse approximation. The relative variance on $\epsilon_{X X}$ is presented on Figure 2. One can note that the filtering rate related to $R_{t}$ on the first derivative is lower than the one related to $R_{x}$. This can be explained from the remark from equations (6), noting that an increase of the span in the direction of the estimated gradient yields a better filtering than in other directions.

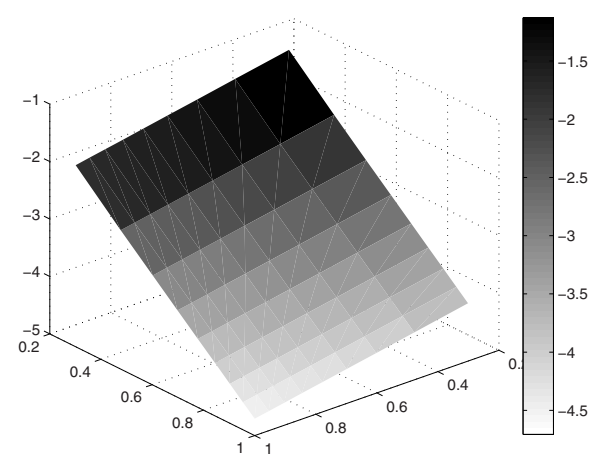

Fig. 2. Relative random error for Space-time Diffuse Approximation

\section{Application to a tensile test on an interlock composite}

\subsection{Detection of local non-linearities - Spatial Diffuse Approximation}

In this section, the $\epsilon_{X X}$ strain field is studied along a traction test on an interlock composite [8] in order to detect the development of local-nonlinearities. The following example is based on a $\pm 45^{\circ}$ traction test on an interlock carbon composite with several loadings and unloadings as described in Figure 3. Digital Image Correlation (DIC) is performed on about 500 images coming from the test, a large number of pictures is thus available. The DIC is performed with CorreliQ4 [9], with a ZOI of 8 pixels. From the ideas proposed in [10], one can define the linear approximation of the strain at each pixel on several parts of the test. As represented on Figure 3, six different parts of the test are chosen for this study, each made up of about 20 snapshots. For each part, the linear response is estimated from the corresponding $N_{k}$ snapshots, $i \in\left\{1, . . N_{k}\right\}, F_{i}$ being the corresponding load, as the slope of the response of each pixel:

$$
\min _{\epsilon_{\text {lin }}^{k}} \sum_{i}^{N_{k}}\left(F_{i} \epsilon_{\text {lin }}^{k}-\epsilon\left(\underline{x}, F_{i}\right)\right)^{2} \quad \text { with, for the } k^{t h} \text { part }
$$

$\epsilon_{\text {lin }}^{k}$ represents the linear approximation field for a load of $1 N$.

These fields are represented on Figure 4 for a span $R$ of 64 pixels for the different studied parts of the test. Considering Figure 4(a), the mean of the linear approximation on the first three parts is ploted, and will be considered as the elastic strain field $\epsilon_{l i n}^{r e f}$ of the specimen, before damage occurs. The other maps are refered by their chronological numbers from Figure 3.

From these fields, it is possible to define a gap to the elastic strain for each part as the discrepancy between the linear approximation related to the part and the reference elastic strain field.

$$
\Delta \epsilon_{\text {lin }}^{k, r e f}(\underline{x})=\epsilon_{\text {lin }}^{k}(\underline{x})-\epsilon_{\text {lin }}^{r e f}(\underline{x})
$$

These $\Delta \epsilon_{\text {lin }}^{k, r e f}$ fields are drawn on Figure 5 for a span $R$ of 64 pixels. Figure 5(a) gives an idea of the effect of the noise, since it compares the third part with the reference, and there should be very 


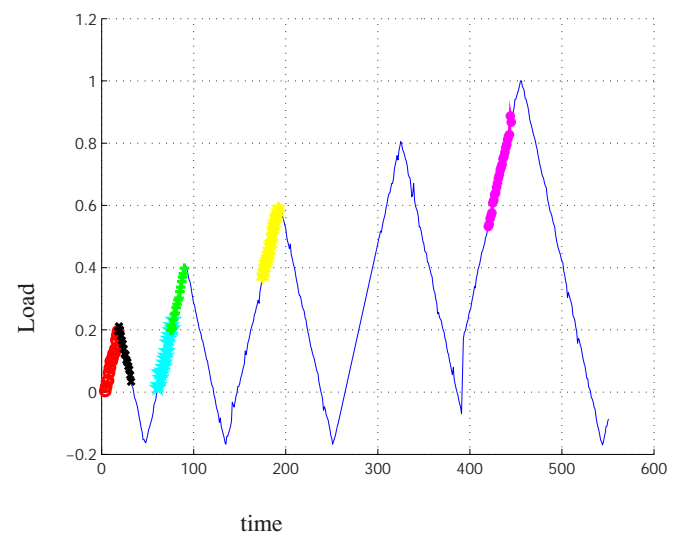

Fig. 3. Loading as a function of the time - the six studied parts

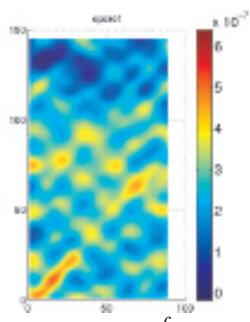

(a) $\epsilon_{\text {lin }}^{r e f}$

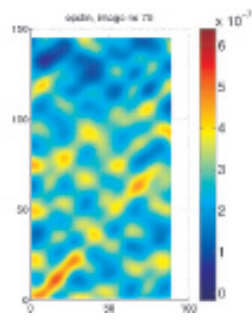

(b) part 4

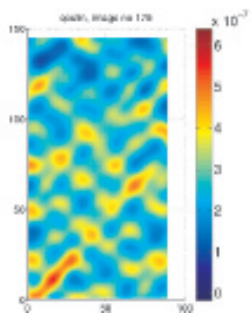

(c) part 5

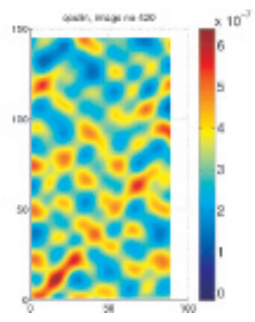

(d) part 6

Fig. 4. Linear approximation of the strain field $\epsilon_{X X}$ for the various parts

little mechanical discrepancy. Its magnitude is lower than on the following maps. One can note that the strain increases (due to local damage) on the upper part of the zone in a first time, inducing an unloading of the central zone. In a second time, it seems, the damage propagates to the whole zone following alternative $\pm 45^{\circ}$ strips damaging and unloading. This tendencie is confirmed by the Figure

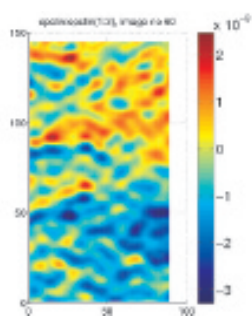

(a) $\Delta \epsilon_{\text {lin }}^{3, r e f}$

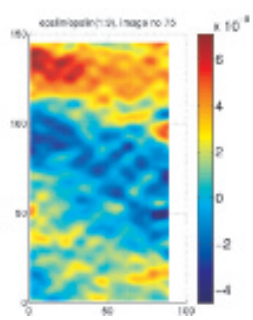

(b) $\Delta \epsilon_{l i n}^{4, r e f}$

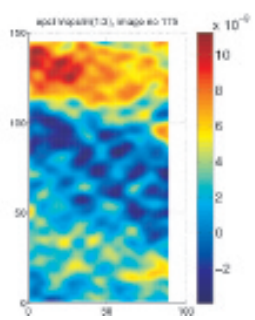

(c) $\Delta \epsilon_{\text {lin }}^{5, r e f}$

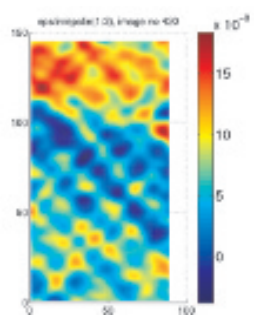

(d) $\Delta \epsilon_{l i n}^{6, r e f}$

Fig. 5. Discrepancy to the elastic strain: $\Delta \epsilon_{\text {lin }}^{k, r e f}$ (10)

6 where the incremental discrepancy is ploted. This discrepancy is defined as:

$$
\Delta \epsilon_{\operatorname{lin}}^{k+1, k}(\underline{x})=\epsilon_{\text {lin }}^{k+1}(\underline{x})-\epsilon_{\text {lin }}^{k}(\underline{x})
$$




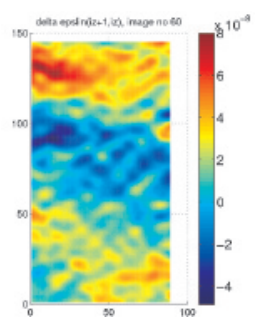

(a) $\Delta \epsilon_{\text {lin }}^{4,3}$

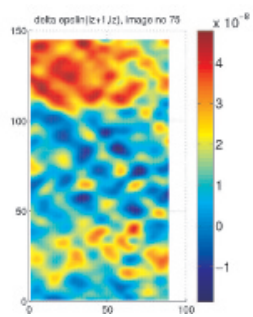

(b) $\Delta \epsilon_{\text {lin }}^{5,4}$

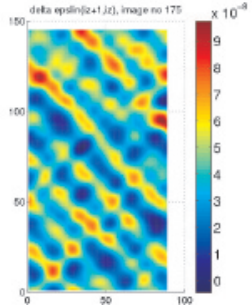

(c) $\Delta \epsilon_{\text {lin }}^{6,5}$

Fig. 6. Incremental discrepancy field: $\Delta \epsilon_{\text {lin }}^{k+1, k}(11)$

Finally, Figure 7 presents the effect of the span $R$ on the discrepancy field $\Delta \epsilon_{\text {lin }}^{5, r e f}$ between the strain coming from part 5 and the reference. On this example, it is obvious that a span $R$ of 64 pixels is too large and some local information is lost. Thanks to the large number of snapshots (about 60) used for the estimate of $\epsilon_{\text {lin }}^{r e f}$, a good filtering of the noise is achieved and one can afford to take a smaller span $R$ to get more detailed local nonlinearities. Nonetheless, Figure 8 shows the same reconstruction with same the color range from the linear strain coming from part 3 alone. It can be seen that small spans leads to strains dominated by the noise. A span $R$ of 64 pixels, if too large, allows at least to find out where the main zones of damage appears, in such an example.

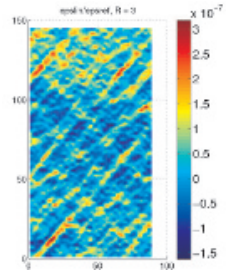

(a) $R=24 p x$

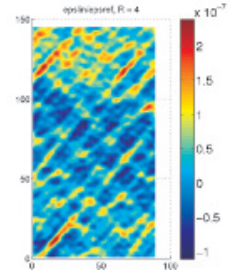

(b) $R=32 p x$

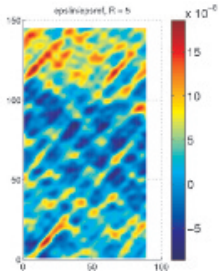

(c) $R=40 p x$

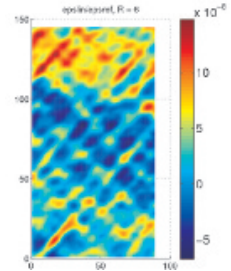

(d) $R=48 p x$

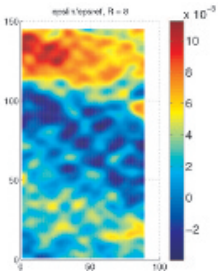

(e) $R=64 p x$

Fig. 7. Discrepancy between part 5 and the reference: $\Delta \epsilon_{\text {lin }}^{5, r e f}$ - effect of the span $R$

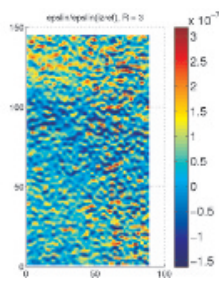

(a) $R=24 p x$

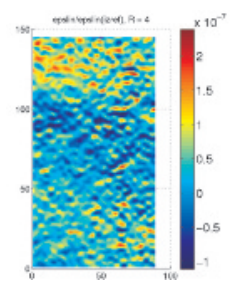

(b) $R=32 p x$

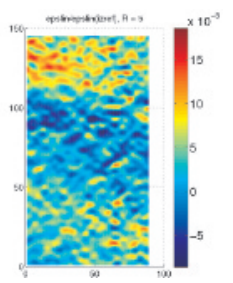

(c) $R=40 p x$

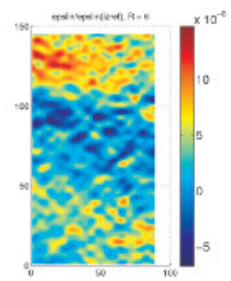

(d) $R=48 p x$

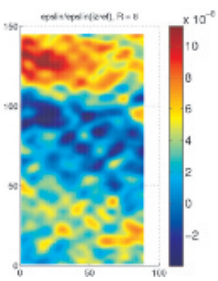

(e) $R=64 p x$

Fig. 8. Discrepancy between part 5 and part 3: $\Delta \epsilon_{\text {lin }}^{5,3}$ - effect of the span $R$

This example suggests that by taking more snapshots one can afford to reduce the span $R$ and therefore reduce the approximation error. To that purpose, the proposed evolution of a Space-Time Diffuse Approximation filtering tool seems appropriate and is to be illustrated on the same test in the following section. 


\subsection{Comparing spatial Diffuse Approximation and Space-time Diffuse Approximation}

From the test described in the previous section, one keeps only the snapshots corresponding to the monotonic loading (leaving the loading-unloading cycles from Figure 3), yielding about one hundred snapshots. One aims at reconstructing the strain field through Space-time Diffuse Approximation. In order to compare the strain field reconstructed here with the linear approximation reconstructed in the previous section, the strain field is divided by the magnitude of the corresponding load.

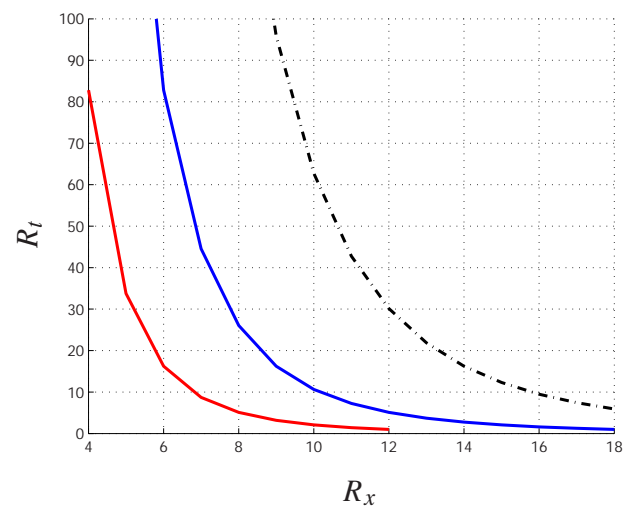

Fig. 9. Isofiltering curves of the Space-time Diffuse Approximation in the $\left(R_{x}, R_{t}\right)$ plan

From the theoretical filtering presented in Section 3, it is possible to introduce isofiltering curves in the $\left(R_{x}, R_{t}\right)$ enabling the same filtering of the noise. For a given level of filtering, one can now tune the time span parameter in order to improve the spatial resolution of the filtering. Sich isofoltering curves are represented Figure 9. One can note that, due to the different behaviour of the spans with respect to the filtering, a given filtering level for a fixed spatial span could lead to a too large time span.

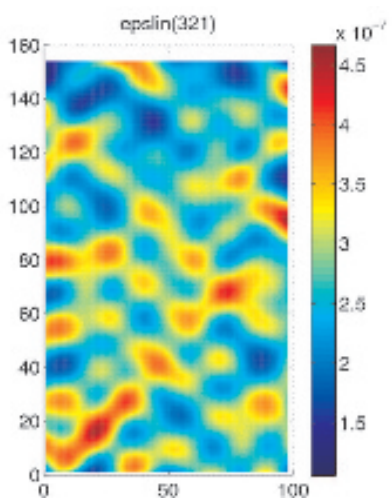

(a) $R_{x}=96 p x, R_{t}=0$

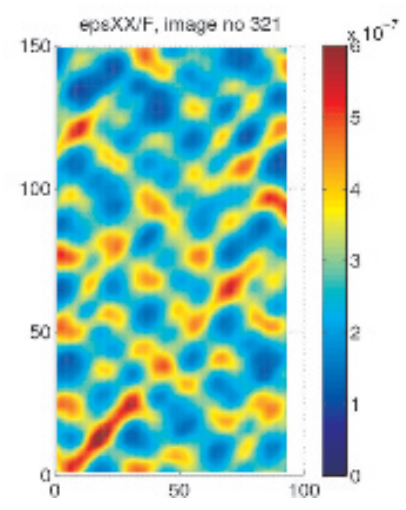

(b) $R_{x}=48 p x, R_{t}=16$

Fig. 10. Two isofiltering reconstructions of $\epsilon_{X X}$

Figure 10 shows the reconstructed strain (divided by the load level) corresponding to a time in the $6^{\text {th }}$ group of Figure 3 (it can therefore be compared to the linear approximation of Figure 4(d)). It is reconstructed for the same filtering level, with both spatial Diffuse Approximation and Space-time Diffuse Approximation. One can note an improvement of the spatial resolution when using the Space-time 
Diffuse Approximation. Furthermore, when comparing the strain field with the linear approximation of Figure 4(d), it seems the Space-time Diffuse Approximation yields a better spatial resolution.

\section{Conclusion}

In this paper, the question of the strain reconstruction from full-field displacement measurements has been adressed. A filtering tool based on the the Diffuse Approximation has been proposed, first treating each snapshot apart. Then, an alternative approach based on a coupled space-time filtering has been proposed in order to take advantage of the great amount of snapshots available on some tests. The effect of the spatial and time spans on the filtering has been studied from a theoretical point of view. The filtering approaches have been successfully applied to the detection of early damage on a tensile test on an interlock composite. The example confirmed the ability of the Space-time Diffuse Approximation to improve the spatial resolution.

\section{References}

1. A. Kobayashi, Handbook on Experimental Mechanics (Wiley, 1993)

2. M. Geers, R. De Borst, and W. Brekelmans, International Journal of Solids and Structures 33-29, (1996) 4293-4207

3. S. Avril, P. Feissel, F. Pierron, P. Villon. Comparison of two approaches for differentiating full-field data in solid mechanics. Measurement Science and Technology, IOP, 21.1,0157032010.

4. Z. Feng and R.E. Rowlands, Computers and Structures 6, (1991) 631-639

5. B. Nayroles, G. Touzot, P. Villon. La méthode des éléments diffus. Comptes rendus de l'Académie des Sciences, série 2, Mécanique, Physique, Chimie, Sciences de l'Univers, Sciences de la Terre, 313-2, 133-138, 1991.

6. W.S. Cleveland, C. Loader Smoothing by local regression: principles and methods, Springer, 1995.

7. S. Avril, P. Feissel, F. Pierron, P. Villon. Estimation of strain field from full-field displacement noisy data. Revue Européenne de Mécanique Numérique, Lavoisier, 17.5-7,857-868 2008.

8. P. Feissel, J. Schneider and Z. Aboura Estimation of the strain field from full-field displacement noisy data: filtering through Diffuse Approximation and application to interlock graphitelepoxy composite, $17^{t} h$ Int. Conference on Composite Materials, 27-31 July 2009, Edinburgh (UK), IOM

9. G. Besnard, F. Hild, S. Roux. Finite-element displacement fields analysis from digital images: Application to Portevin-Le Châtelier bands. Experimental Techniques, 46, 789-803, 2006.

10. F Pierron, B. Green and M R Wisnom. Full-field assessment of the damage process of laminated composite open-hole tensile specimens. Part II: Experimental results

Composites Part A: Applied Science and Manufacturing, 38-11,2321-2332 2007. 